\title{
A Group Based Point-To-Multipoint MBMS Algorithm over the HSDPA Network
}

\author{
Ahsan Chaudhry \\ School of Electrical Engineering and Computer \\ Science, The University of Newcastle, Callaghan, \\ NSW, 2308, Australia \\ ahsan.chaudhry@studentmail.newcastle.edu.au
}

\author{
Jamil Y. Khan \\ School of Electrical Engineering and Computer \\ Science, The University of Newcastle, Callaghan, \\ NSW, 2308, Australia \\ Jamil.khan@newcastle.edu.au
}

\begin{abstract}
Multimedia Broadcast and Multicast Service (MBMS) specification was introduced in the release 6 of the 3GPP standard to deliver multimedia content to a large group of users in the UMTS network. With the MBMS system generally the same content is transmitted to multiple users in a unidirectional fashion, typically by multiple base stations to improve the coverage over a large geographical area. From the radio network point of view the standard supports the point-to-point (p-t-p) and the point-to-multipoint (p-t-m) transmission modes. In this paper we propose a group based $p-t-m$ MBMS algorithm utilising the scalable video coding techniques to improve overall QoS of multimedia services. The group based MBMS algorithm has been proposed to support moderate to high date rate multicasting services over the HSDPA link. The proposed algorithm has been simulated using an OPNET simulation model. Initial results show that the proposed algorithm could offer higher data and improved QoS for MBMS users.
\end{abstract}

\section{INTRODUCTION}

The Multimedia Broadcast Multicast Service (MBMS) standard which was proposed in the release 6 of the 3GPP standard can support efficient multicasting and broadcasting services over the UMTS network [1].The MBMS standard is overlayed on the existing UMTS network to reduce the cost of the system development. Hence, it is possible for MBMS service providers to take advantage of the increasing data rate of the HSPA (High Speed Packet Access) system to introduce diverse and high quality multimedia contents. The standard supports both point-to-point ( $\mathrm{p}-\mathrm{t}-\mathrm{p}$ ) and point-to-multipoint ( $\mathrm{p}$ $\mathrm{t}-\mathrm{m}$ ) transmission modes. The $\mathrm{p}-\mathrm{t}-\mathrm{p}$ mode is more suitable for small number of users whereas the p-t-m mode is more suitable for large number of users. Since, the MBMS is overlayed on top of the UMTS network the network can automatically take advantage of the network point to point channels to carry service level feedback from users. The quality of MBMS services in a network will depend on radio conditions and cellular network design. To support MBMS services macro diversity was proposed where combining transmissions of the same content from multiple base stations provide a significant diversity gain. The macro diversity was proposed to avoid relaying feedback from users which would consume a large amount of bandwidth on the uplink [2]. Such approach could improve the coverage area and offer a reduction of 4-6 $\mathrm{dB}$ transmission power compared to a single reception only. However, the drawback of the system is use of higher radio resources because the same content is transmitted via multiple base stations. In this paper we propose an improved MBMS QoS and service coverage technique based on single Node-B solution. Using the approach multicast group receivers are separated into groups where the scalable video coders are used to receive multimedia contents. The proposed algorithm is implemented on the High Speed Downlink Packet Access (HSDPA) standard [3].

To develop the proposed algorithm we assume that future multimedia content can be generated using scalable video coders where a significant amount of bandwidth is consumed by video contents. In a wireless network to receive high quality multimedia content it is necessary to have a high data rate link. High data rate links can only be allocated to users if the link can maintain sufficient SNR value. Maintaining high SNR throughout a cell is extremely difficult and this factor is one of the bottlenecks of wireless systems. In this work we examined a HSDPA based MBMS system where multimedia services could be provided without use of macro diversity. We combine the concept of group based multicasting and scalable video coding technique to improve the overall QoS of a MBMS system. A scalable video coder can create multilayered coded video stream to send video contents by generating an array of data packets [4]. The selectable nature of the codec will allow receivers to select number of layers it wants to receive. In a wireless network a receiver can select the number of video layers based on their available transmission link rate. Without the macro diversity it is well known that data rates towards cell edges will always be lower compared to data rates around the vicinity of a base station. On the other hand if multicast user group is formed by combining users from different locations of a cell using standard video coders to receive multimedia content then the variability of the transmission link quality will constrain the achievable data rate of the user. If users select their video coding rate based on their link qualities then a number of multicast groups can be formed where users can select their video coding rates based on their transmission link qualities and can improve the overall QoS of the system.

Our proposed group based multicasting algorithm has been developed on the HSDPA standard. As one of the initial steps of the 3GPP Long Term Evolution (LTE) plan, the HSDPA standard has been introduced to significantly improve downlink capacity over next few years. The HSDPA architecture was initially introduced in the 3GPP release 5. The HSDPA channel uses a short $2 \mathrm{~ms}$ transmission time interval (TTI), and uses variable coding and modulation techniques to achieve high data rate. In addition to above techniques the node-B based fast scheduling and fast physical layer hybrid automatic repeat request (H-ARQ) techniques are used in the HSDPA standard. The Standard supports a fast channel- 
dependent scheduling to allocate shared channel at any particular moment considering the instantaneous radio channel conditions. According to the HSDPA roadmap within the next year the air interface data rates could reach up to $28.8 \mathrm{Mbps}$ and eventually the data rate could reach $100 \mathrm{Mbs}$ under the LTE plan. This makes HSDPA an ideal platform to implement future MBMS services.

The paper is organised as follows. Section II reviews several relevant papers on HSDPA based MBMS services. Section III describes our proposed group based multicast transmission technique using scalable video coding technique. Simulation model and parameters are described in the section IV. Some initial simulation results are presented in the section $\mathrm{V}$ and a brief conclusion is presented in the section VI.

\section{HSDPA BASED MBMS}

With the advancement of the HSDPA standard, research community have shown a lot of interest in exploring its adaptive high data rate capability to improve system throughput and QoS for different Real Time and Non-Real Time Applications. MBMS is considered to be one of the services which could generate significant revenues for mobile operators and therefore has been investigated for improving user perceived QoS of multimedia applications using the HSDPA standard. The MBMS can be delivered using either $\mathrm{p}-$ $t-p$ or $p-t-m$ mode of transmission. Capability for transmitting MBMS over HSDPA in p-t-p mode has been studied in the reference [5] and authors have presented a fair channeldependent scheduler. The paper concluded that for strict delay and jitter requirements of streaming video traffic, HSDPA can support up to 15 users per tri-sector cell, serving more users decreases capacity and increases the delay. It is also proposed that the HSDPA could be used as an additional p-t-p repair mechanism for recovering lost packets instead of standalone operation to support MBMS services. In the reference [6] different multi-resolution schemes have been proposed to improve the MBMS performance. One of the proposals is the use of HSDPA as a multi-resolution technique to achieve different data rates based on the reported feedback. Results show improvement in average system throughput and authors have found it to be "an interesting option". However, the technique only considers HSDPA p-t-p mode and therefore not being able to serve large number of users. The reference [7] considered the HSDPA p-t-m mode and looked into the coverage of MBMS with different fixed number of retransmissions, and Modulation and Coding Sets (MCS). Results showed that for all combinations 1 to 4 retransmissions are required to achieve above $95 \%$ coverage. Since the retransmissions uses additional radio resources, the goodput decreases with the number of retransmissions. Authors also looked into the adaptive retransmissions issues based on UE feedbacks and showed the improvement of transmission resource utilisation by up to $70 \%$ is possible. Although not directly mentioned but it is still implied that any number of retransmission including the adaptive feedback mechanism reduces the overall goodput.

\section{GROUP BASED MULTICAST TRANSMISSION ALGORITHM}

We propose a group based transmission algorithm to support MBMS services over the HSDPA network. An HSDPA connection adaptively set its transmission rate based on the Channel Quality Indicator (CQI) value obtained via the user feedback. To serve large population of multimedia users in a cell it is unrealistic to expect that all users can receive same data rate and hence it is realistic to expect that received data rate for different users will be different. In order to implement acceptable multimedia services we propose a group based transmission algorithm which takes advantage of scalable video coding technique. We assume that each user will be able to decode its video content based on its transmission condition. Under our proposed scheme all MBMS users will report their measured CQI value at the time of a call setup. Based on the feedback the Node-B will form a number of groups who will receive multimedia content adjusted according to their transmission condition. The scheme is a hybrid of HSDPA, the $p-t-p$ and the $p-t-m$ modes. The $p$ - $t-p$ mode schedules individual users transmission based of the reported CQI whereas in the $\mathrm{p}-\mathrm{t}-\mathrm{m}$ mode users receive the same channel but provide no feedback. The algorithm uses the Adaptive Modulation and Coding $(A M C)$ mechanism of the HSDPA standard at the group level rather than at the individual user level. The algorithm divides all UEs into groups based on their CQI values using the following min CQI technique which is shown in equation 1. A group CQI is selected based on the minimum CQI values of group members. Each group is then served with the HS-DSCH channel using appropriate number of codes.

$\operatorname{Group}\{C Q I\}=\operatorname{Min}\left[C Q I_{i}\right], i \in N$

After the formation of groups of UEs each group receives their multimedia content via the HSDPA link using the code multiplexing technique i.e. codes are multiplexed for each group in every TTI (Transmit Time Interval) of $2 \mathrm{~ms}$. The grouping mechanism improves the overall QoS of the system because groups with higher CQI values will not be constrained by the lower CQI UEs thus improving the total QoS as well as the coverage area. The number of groups can be optimised based on the user requirements and application data rates. Using the above technique users with higher CQI values can receive high quality video contents and will not be affected by the presence of other multicast UEs. This technique does not require constant feedback. A UE will only report change of CQI values when the measured CQI is higher or lower for a numbers of subsequent TTIs. Once a UE reports a different $\mathrm{CQI}$ value the node-B could move the UE to a different group to receive multimedia contents at an appropriate rate. The approach is scalable and requires minimum signalling on the Uplink.

\section{SIMULATION MODELING}

A discrete-time event-driven simulation model was developed based on the OPNET Modeler package to analyse the performance of the proposed algorithm. The transmission 
channel model includes path loss, shadow and multipath fading models. The path loss was modelled using COST 231 Hata Model. The shadow fading was modelled using the lognormal distribution and the correlation distance. The multipath fading was modelled using the $3 \mathrm{GPP}$ Pedestrian A $(3 \mathrm{~km} / \mathrm{h})$ model using the tapped delay line generating random number of taps and combing them to generate the loss in each TTI. The Signal to Interference and Noise Ratio (SINR) value was calculated at the output of the receiver using the equation 2 .

$$
S I N R=\frac{P_{H S-D S C H}}{P_{\text {own }}\left[(1-\alpha)+G^{-1}\right]}
$$

Where $P_{H S-D S C H}$ is power allocated to the High Speed Downlink Shared Channel (HS-DSCH), $P_{\text {Own }}$ is total Node-B power, $\alpha$ is the orthogonality factor and $\mathrm{G}$ is the geometry factor given by the equation 3 .

$$
G=\frac{P_{\text {own }}}{P_{\text {other }}+N}
$$

$P_{\text {other }}$ is the total interference generated by other cells and $N$ is the thermal Noise.

TABLE I. HSDPA SINR Targets for different CQI
\begin{tabular}{|c|c|c|}
\hline CQI & Bit Rate (Kb/s) & SINR (dB) \\
\hline 1 & 68.5 & -7.87 \\
\hline 2 & 86.5 & -6.58 \\
\hline 3 & 116.5 & -5.24 \\
\hline 4 & 158 & -4.49 \\
\hline 5 & 188.5 & -3.62 \\
\hline 6 & 230.5 & -1.3 \\
\hline 7 & 325 & -0.44 \\
\hline 8 & 396 & 0.44 \\
\hline 9 & 465.5 & 1.46 \\
\hline 10 & 631 & 2.16 \\
\hline 11 & 741.5 & 3.32 \\
\hline
\end{tabular}

An Iterative Solution Coded Modulation Library (ISCML) based on the open source toolbox is used to run the link level simulations in Matlab to obtain the Signal to Interference and Noise Ratio (SINR) values [8]. The simulator takes into account the channel conditions, interleaving, channel coding, receiver structure and decoding to predict the Block Error Rate (BLER) of the receiver. These values were derived for MBMS services corresponding to $1 \%$ BLER for different CQI values. Table I lists transmission bit rate and SINR values for different CQI values. Category 7 and 8 UEs were considered for our simulations. Initial simulations were run with 50 UEs in three scenarios. Scenario 1 represents uniform distribution of UEs in the cell i.e. the UEs are uniformly spread across the whole cell. Scenario 2 and 3 represent the random distribution of UEs across the cell. Linear congruential pseudorandom number generator is used to derive the random position coordinates of UEs from the BS with different seed values both of scenario 2 and 3 . In the initial simulation model no mobility was introduced. In our simulation we consider that a UE is inside an MBMS coverage area if the UE receives information with a
BLER of $1 \%$ or less. UE with BLER of more than $1 \%$ it is considered to be out of coverage. Key simulation parameters are listed in the Table II.

\begin{tabular}{|c|c|}
\hline Parameter & Value \\
\hline No. of Node-B & 1 \\
\hline Antenna type & Omnidirectional \\
\hline Cell Radius & $933 \mathrm{~m}, 500 \mathrm{~m}$ \\
\hline BS Antenna gain + cable loss & $15 \mathrm{~dB}$ \\
\hline BS Total Transmit Power & $12 \mathrm{~W}$ \\
\hline HSDPA Used Power & $7 \mathrm{~W}$ \\
\hline Orthogonality Factor $(\alpha)$ & $0.6,0.8$ \\
\hline Geometry Factor (G) & $-3-10 \mathrm{~dB}$ \\
\hline Maximum no. of codes & 3 \\
\hline Simulation time & $120 \mathrm{sec}$ \\
\hline MBMS Sessions & 1 \\
\hline HARQ Type & Chase Combining \\
\hline Link adaptation Algorithm & Standard AMC \\
\hline Total no. of UEs/cell & 50 \\
\hline
\end{tabular}

TABLE II. Simulation Parameters

V. SIMULATION RESULTS

In this section we analyse various simulation results obtained using the OPNET model. All the results gathered from above described model are using stationary UEs i.e. UEs remain in a fixed location during a MBMS session. First, we analyse the performance of our proposed algorithm for different traffic distributions and transmission conditions and then we compare our algorithm with conventional HSDPA based MBMS systems. Fig. 1 shows the MBMS coverage distribution areas for different transmission data rates for $933 \mathrm{~m}$ and $500 \mathrm{~m}$ cell sizes. To obtain this result we used a single/non-group based transmission and run the simulation for different CQI values. As shown on the figure that if we require higher coverage areas for MBMS services then the transmission data rate will be restricted by lowest CQI UEs in the cell. The coverage drops almost linearly with the increasing data rate. For randomly distributed mobiles the coverage for high data rate is slightly better than the uniform case. The coverage drops to only $12 \%$ at highest achievable data rate of $396 \mathrm{kbps}$ for $933 \mathrm{~m}$ cell size. Same coverage pattern can be observed for a $500 \mathrm{~m}$ cell size. The sudden drop of coverage for the Uniform distribution of UE is due to the reason that almost $38 \%$ UEs are placed near the cell edge and therefore they can not receive higher data rates. In a practical scenario we expect that UEs will be quite often randomly distributed. This simulation did not include the HARQ option.

Next we examine the effect of the HARQ on the MBMS service coverage based on for different CQI values. Fig. 2 compares the coverage distribution for uniform distribution of UEs with no and single retransmission. The graph shows that there is some improvement in coverage due to retransmission but this comes at a cost of decreased goodput. The goodput drops due to the fact that additional TTIs are used to support the retransmission to some users which results in lowering the goodput. It is also noticeable that no or little improvement in coverage can be achieved for higher CQI values. This is 
because higher data rates require multiple retransmissions to get the packet correctly to UE. For the above reason retransmissions were not considered for further simulations.

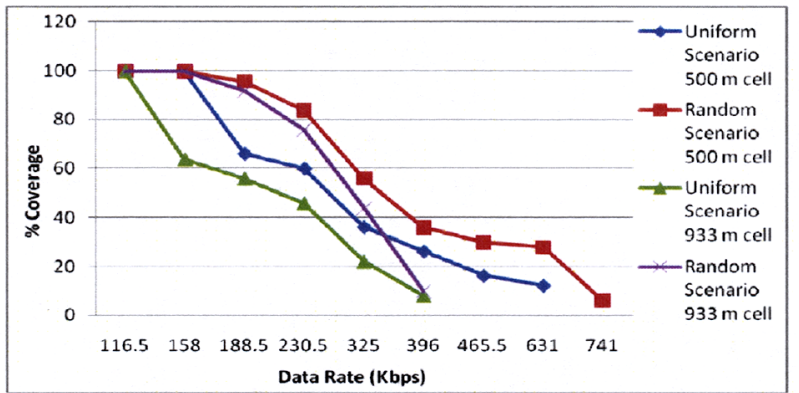

Figure 1. Distribution of coverage areas for non-group based MBMS transmission scheme.

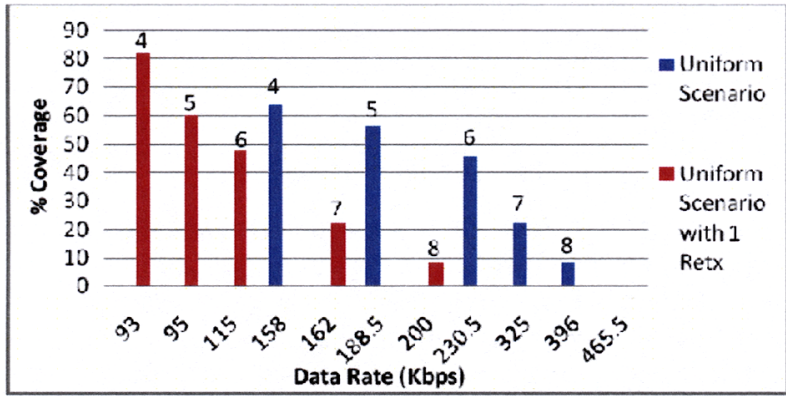

Figure 2. MBMS service coverage distribution with and without retransmissions

Fig. 3 and Fig. 4 shows the distribution of reported CQI by UEs for $933 \mathrm{~m}$ and $500 \mathrm{~m}$ cell sizes respectively. For the 933 $\mathrm{m}$ cell around $36 \%$ of UEs in the uniform scenario and only $4 \%$ UEs in the random scenario have reported CQI 3, the lowest CQI value. The uniform distribution shows two large groups of UEs being able to receive CQI values of 3 and 6. For the $500 \mathrm{~m}$ cell size the CQI values are also uniformly distributed in two groups. For example, CQI values of 4 and 6 should be able to cover most of the UE. It is worthwhile to mention here that a UE with a higher reported CQI value will be able to receive transmissions with lower CQI but can not receive transmissions of higher CQI. In order to achieve maximum coverage in a group based transmission scheme we select group CQI values based on the lowest value of reported CQI. For the $933 \mathrm{~m}$ cell size, if we use two group based transmissions then the logical choice of CQIs will be 3 and 6 for uniformly distributed UEs. In this case UEs who are reporting CQI 3-5 will join the group 1 and receive the lower data rate of $116.5 \mathrm{kbs}$, and the rest of the group receive higher data rate of $230.5 \mathrm{kbs}$. In this case about $50 \%$ UEs will receive higher data rate compared to a single group/non-group based transmission. Fig. 5 shows the achievable data rate for a single group/non-group based transmission scheme coverage. Single group/non-group means that all the UEs fall under one group and received data rate corresponds to the lowest reported CQI value. Fig. 6 shows the coverage areas for a two group based transmission scheme. The figure shows that for all scenarios the overall transmission rate has improved. For the random 2 scenario about $80 \%$ of UEs will be able to receive their services at $230.5 \mathrm{kbs}$ as opposed to $158 \mathrm{kbs}$ for a single group/non-group based transmission. Similarly the uniform distribution shows about $50 \%$ users will receive better data rate. Figure 7 shows the achievable data rates for same scenarios when three groups are used for transmission. This result shows further improvements in terms of data rates which justifies using the group based transmission schemes combining with scalable video coders to deliver multimedia contents.

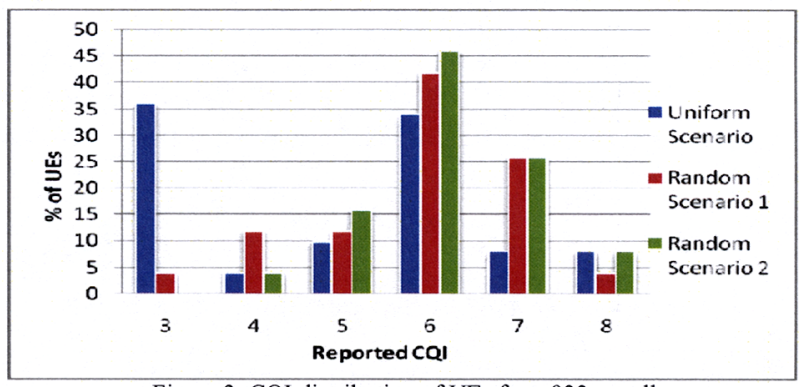

Figure 3. CQI distribution of UEs for a $933 \mathrm{~m}$ cell

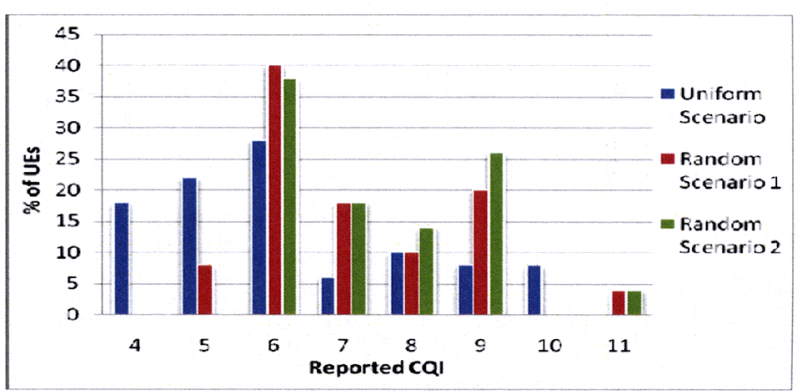

Figure 4. CQI distribution of UEs for a $500 \mathrm{~m}$ cell

Further simulation results are presented for the $500 \mathrm{~m}$ cell size in Fig. 8 and 9. Simulation results show the similar trends. Also, results show that for smaller cell sizes considerable high data rates are achievable for MBMS services. Non group based transmission offers maximum of $230.5 \mathrm{kbs}$ services with a $100 \%$ coverage whereas Fig. 9 shows that three group based approach can extend the data rate to $465.5 \mathrm{kbs}$ to about $30 \%$ UEs.

Simulation results clearly indicates that signifcant QoS improvments can be achieved for MBMS services when the group based transmission schemes are used. As mentioned earlier that the proposed algorithm does not require too much feedback to manage these groups. When a UE intiates a session it reports its CQI value and the next time it reports only when the CQI values have changed significantly so that it can move to a different group. For example, in a $933 \mathrm{~m}$ cell for uniform distribution of traffic UEs with CQI values of 3, 4 and 5 will be combined in a single group. If any UE within the group find variation of its own CQI but within the group range then it will not report the CQI value. When the CQI value become 6 or higher then it can report the value with a request to change the group if the application demands. For a 
stationary UE environment it is unlikely that reported CQI value will change duing MBMS session because the SNR value remains reasonable steady most of the time. It is also likely that in most future wireless networks large proportin of nodes will be stationary. Hence, it can be seen that the proposed algorithm not only introduces lower signalling rate but also offers scalability feature.

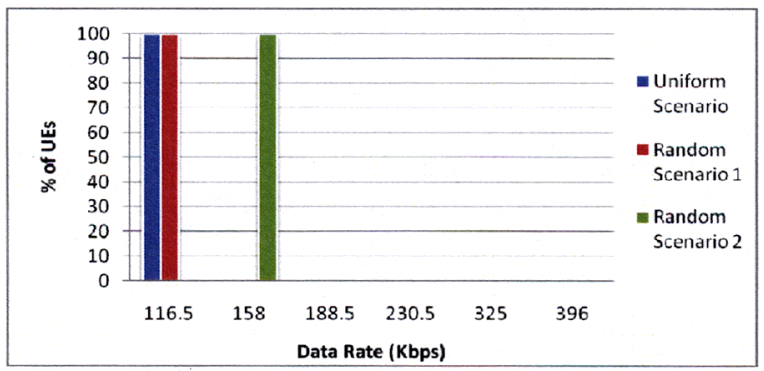

Figure 5. Single group/non-group based achievable data rates and MBMS coverage areas for the $933 \mathrm{~m}$ cell.

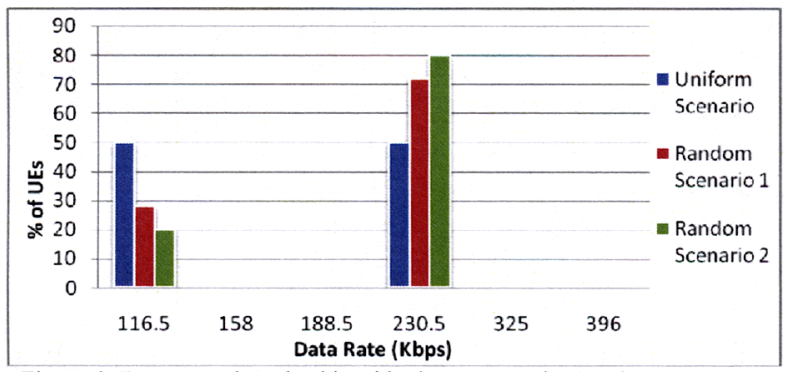

Figure 6. Two group based achievable data rates and MBMS coverage areas for the $933 \mathrm{~m}$ cell.

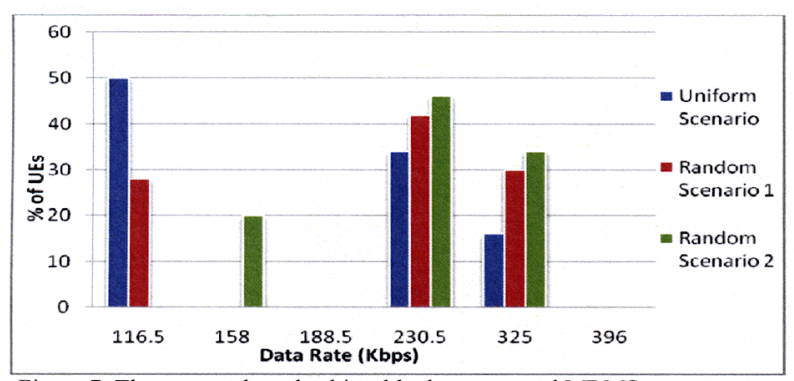

Figure 7. Three group based achievable data rates and MBMS coverage areas for the $933 \mathrm{~m}$ cell.

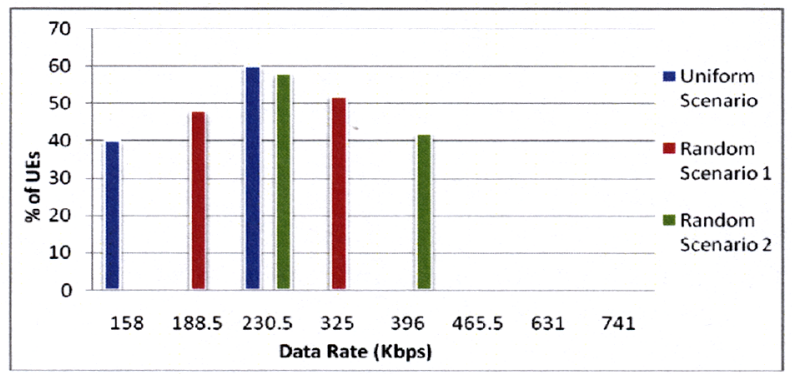

Figure 8 . Two group based achievable data rate by and MBMS coverage areas for the $500 \mathrm{~m}$ cell.

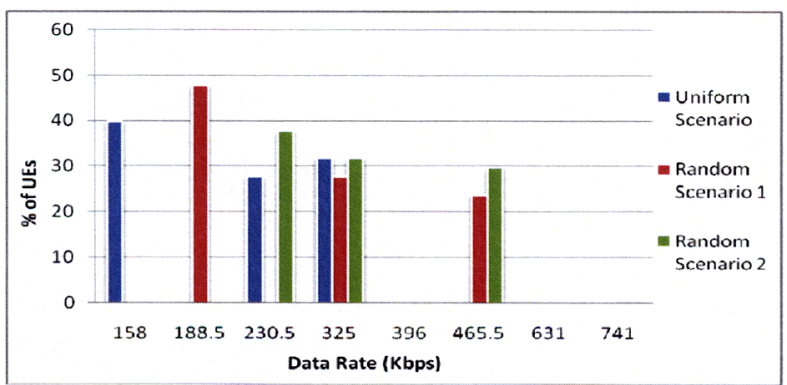

Figure 9. Three group based achievable data rate and MBMS coverage areas for the $500 \mathrm{~m}$ cell.

\section{CONCLUSION AND FUTURE WORK}

The paper presented an efficient group based MBMS performance enhancement scheme. Simulation results show that the proposed scheme offer signification improvements of MBMS service QoS when combined with a scalable or a hierarchical video coder. Currently we are focusing on the introduction of mobility among UEs which will require developing an efficient group management scheme to reorganise groups as UEs move from location to location. Mobility of UEs will introduce a serious group management problem because it is possible that quite often UEs will move to different location thus introducing a new connection management issue.

\section{REFERENCES}

[1] "MBMS Architecture and functional description, Technical realization (stage 2)," 3GPP TSG TS23.246, Rev. v6.1.0, December 2003.

[2] S. Parkvall, E. Englund, M. Lundevall, and J. Torsner, "Evolving 36 Mobile Systems: Broadband and Broadcast Services in WCDMA," in IEEE Communications Magazine. vol. 44, February 2006, pp. 68 - 74.

[3] "High Speed Downlin Packet Access (HSDPA) Stage 2," 3GPP TS 25.308 v5.4.0 Release 6, March 2004.

[4] A. Asif, U. T. Nguyen, and G. Xu, "Scalable Video Multicast over MANETs," in 8th IEEE Workshop on Multimedia Signal Processing, 2006, pp. 403408.

[5] F. Leitao and A. Correia, "HSDPA Delivering MBMS Video Streaming," in 8th International Symposium on Wireless Personal Multimedia Communications, Aalborg, Denmark, 2005, pp. 1538 - 1542.

[6] A. M. C., J. C. M. Silva, N. M. B. Souto, L. A. C. Silva, A. B. Boal, and A. B. Soares, "Multi-Resolution Broadcast/Multicast Systems for MBMS," IEEE Transactions on Broadcasting, vol. 53, pp. 224 - 234, March 2007.

[7] V. Vartiainen and J. Kurjenniemi, "Point-to-Multipoint Multimedia Broadcast Multicast Service (MBMS) Perfromance Over HSDPA," in The 18th Annual IEEE International Symposium on Personal, Indoor adn Mobile Radio Communications (PIMRCO7), Athens, Greece, 2007.

[8] M. C. Valenti, "Interactive Solutions Coded Modulation Library http://www.iterativesolutions.com/Matlab.htm." vol. 2007. 